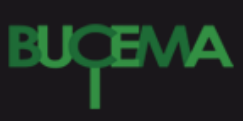

Bulletin du centre d'études médiévales d'Auxerre | BUCEMA

Hors-série $n^{\circ} 3 \mid 2010$

Présentation et mise en valeur des sites archéologiques religieux en milieu urbain

\title{
Le site de La Charité-sur-Loire - Exposé des résultats scientifiques
}

Chantal Arnaud

\section{(2) OpenEdition}

Journals

Édition électronique

URL : https://journals.openedition.org/cem/11386

DOI : $10.4000 /$ cem. 11386

ISSN : 1954-3093

Éditeur

Centre d'études médiévales Saint-Germain d'Auxerre

Référence électronique

Chantal Arnaud, "Le site de La Charité-sur-Loire - Exposé des résultats scientifiques », Bulletin du centre d'études médiévales d'Auxerre | BUCEMA [En ligne], Hors-série n 3 | 2010, mis en ligne le 15 mars 2010, consulté le 03 mars 2023. URL : http://journals.openedition.org/cem/11386 ; DOI : https:// doi.org/10.4000/cem. 11386

Ce document a été généré automatiquement le 3 mars 2023.

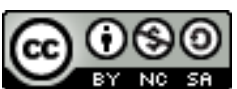

Creative Commons - Attribution - Pas d'Utilisation Commerciale - Partage dans les Mêmes Conditions 4.0 International - CC BY-NC-SA 4.0

https://creativecommons.org/licenses/by-nc-sa/4.0/ 


\title{
Le site de La Charité-sur-Loire - Exposé des résultats scientifiques
}

\author{
Chantal Arnaud
}

\section{Historique des recherches archéologiques à La Charité-sur-Loire}

1 Le site de La Charité-sur-Loire est essentiellement connu pour son église Notre-Dame, ancienne prieurale d'un monastère dépendant de l'abbaye de Cluny. Cet intérêt a été suscité notamment lors de la découverte en 1815 d'un tympan sculpté du XII ${ }^{\mathrm{e}}$ siècle pris dans une maison accolée à la façade de l'église, puis lorsque, en 1837, Prosper Mérimée s'oppose à la destruction d'une partie de l'édifice, celle située entre le clocher et la partie utilisée comme église, destruction envisagée afin de faire passer la route royale. L'église est alors classée Monument historique sur la première liste de 1840. Depuis, historiens et historiens d'art n'ont cessé de donner une place de choix à cet édifice dans les manuels d'histoire médiévale. 


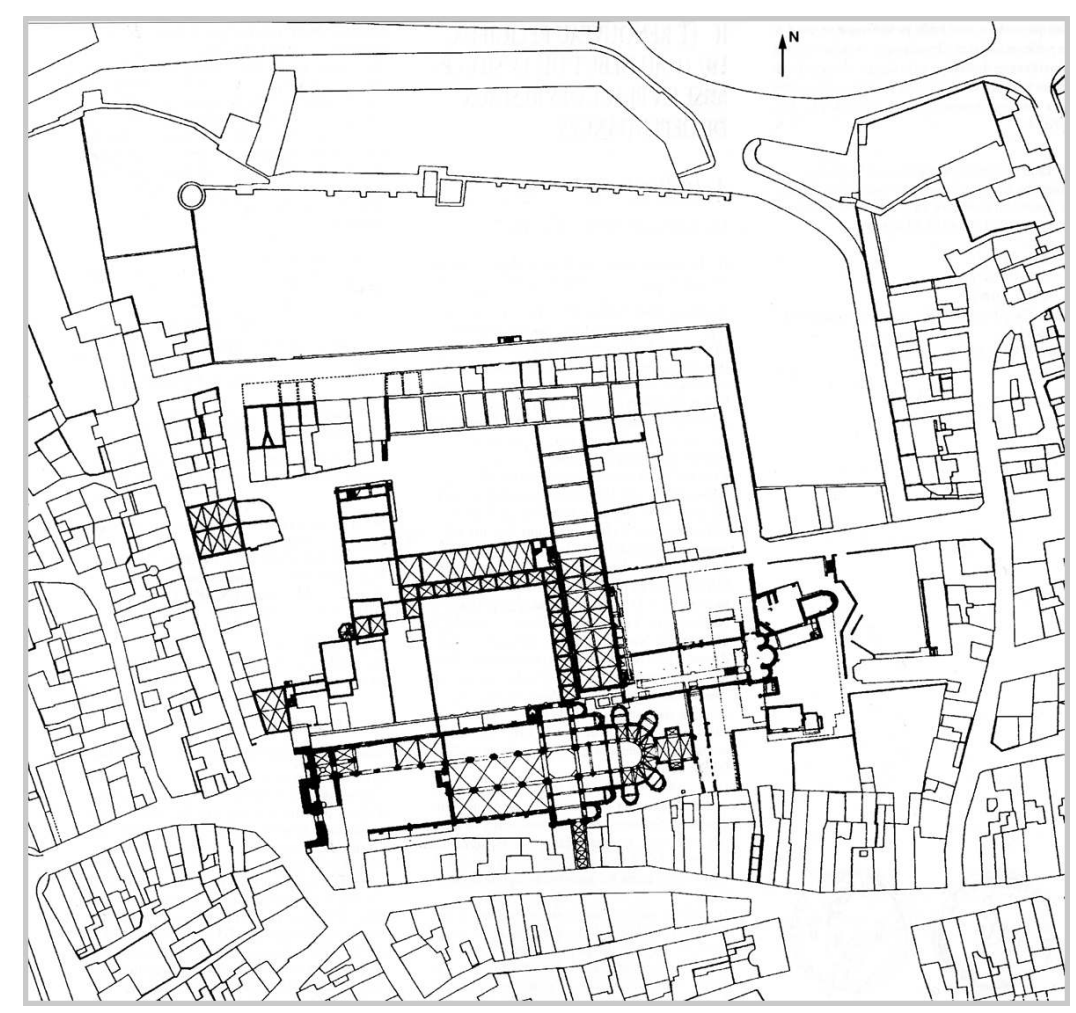

Fig. 1 - Plan du prieuré de La Charité-sur-Loire.

2 Aussi, avant 1974, les « interventions archéologiques » ne se situaient-elles qu'en lien avec cet édifice. Grâce au dépouillement des journaux locaux, notamment l'Écho de La Charité et Le Charitois, on peut faire remonter les premières découvertes archéologiques au début du xxe siècle : en 1904, un groupe d'élèves de l'École des chartes découvre un caveau à l'intérieur de l'église ; cinquante ans plus tard, à la demande des Bâtiments de France puis des Beaux-Arts, différents sondages sont réalisés dans la nef pour retrouver le sol médiéval et les divisions internes de l'église; en 1962, sont dégagés les vestiges de l'ancienne abside du premier collatéral nord appartenant à l'édifice du $\mathrm{xI}^{\mathrm{e}}$ siècle et démolie lors de la construction du déambulatoire au XII siècle ; en 1971, à l'occasion du dégagement des habitations accolées contre le mur gouttereau sud, est mis, entre autres, au jour une sépulture en coffre maçonnée.

En 1974, la municipalité de La Charité souhaite construire un centre médico-social sur un espace de jardin situé au nord-est du chevet de cette église. Des carottages du soussol sont effectués et laissent supposer la présence de vestiges construits. L'année suivante lors des terrassements, le chevet d'une église est mis au jour : les travaux sont stoppés et un sauvetage archéologique est entrepris. La ville décide de construire le centre médico-social ailleurs et d'aménager cet espace en jardin archéologique.

4 Jusqu'en 1982, se succèdent sauvetages archéologiques, prospections et fouilles programmées sous la responsabilité de Serge Renimel: les travaux se concentrent surtout autour du chevet de cette nouvelle église. Les recherches dans les sources d'archives mettent en valeur l'existence d'une église connue sous le vocable de SaintLaurent, au côté de celle de Notre-Dame.

En 1989, une étude du potentiel archéologique est réalisée par V. Deloffre-Roumégoux ; elle atteste l'importance et la qualité des sépultures qui sont situées au sud de cette église. 
6 Pour un large public, ces travaux de recherche restent sans lendemain. Ils concernent un petit secteur de la ville par rapport à l'ensemble du prieuré. On s'interroge aussi sur la valeur de ces ruines au regard de la qualité architecturale de l'église Notre-Dame.

7 En 1990, l'ensemble prieural de La Charité est désigné «Grand site culturel et touristique » créant un nouveau dynamisme pour la mise en valeur de l'ensemble du site. Grâce à la volonté des responsables administratifs et à l'appui des chercheurs, de nouvelles perspectives de recherches archéologiques peuvent être envisagées.

De 1991 à 1995, les interventions archéologiques sont essentiellement réalisées dans le cadre des études préalables des Monuments historiques. Elles permettent de renouveler totalement l'approche du site.

9 Par la suite, les interventions sont surtout liées aux différentes phases d'aménagement des Monuments historiques; elles apportent également leur pierre à une meilleure compréhension du site. La principale fouille a lieu en 2003 sous la responsabilité de David Billoin, archéologue INRAP, dans le secteur de la galerie située entre les deux églises.

\section{Les résultats scientifiques}

10 L'histoire de ce prieuré clunisien est relativement bien connue. L'acte de fondation du prieuré est daté de 1059 : il est stipulé la donation de la terre à Cluny pour y fonder une dépendance monastique. En 1107, l'église Notre-Dame est consacrée par le pape Pascal II lors de son passage en France. Quelques incendies et de nombreux travaux ponctuent l'histoire médiévale de ce lieu. L'incendie de 1559 marque une étape importante dans l'évolution topographique du prieuré car il en détruit une bonne partie créant deux nouveaux espaces "vides" de constructions : sont aménagés un vaste jardin au chevet de Notre-Dame appelé « square des bénédictins » et une nouvelle place entre ce qui reste de la nef de Notre-Dame et de l'ancienne façade.

\section{Les recherches jusqu'en 1991}

11 Avec la découverte du chevet de l'église Saint-Laurent en 1974, les études scientifiques qui sont menées entre 1975 et 1989 se concentrent uniquement sur ce secteur de la ville : elles posent comme postulat de base d'être en présence d'un premier monastère de taille plus réduite qui a vu le jour avant la mise en place de l'imposante église NotreDame et des ses dépendances.

$12 \mathrm{Au}$ chevet de l'église Saint-Laurent, la mise au jour de tout un ensemble de constructions ayant servi d'hôtellerie, de cuisine et d'atelier témoigne d'une occupation dense de cet espace pendant toute la période médiévale. Le matériel découvert à l'intérieur et autour de ces bâtiments apporte une connaissance nouvelle, pour l'époque, sur l'art de vivre au Moyen Âge.

13 Du côté sud, l'espace est réservé aux inhumations: les quelques sépultures étudiées attestent l'importance et la qualité de leur mise en œuvre ; les plus anciennes datent du $\mathrm{XI}^{\mathrm{e}}$ siècle et concernent des individus masculins; mais dès la deuxième phase d'inhumations, ces dernières concernent une population de tout sexe et de tout âge et ce jusqu'au Xvi 'e siècle. 
On imagine alors l'église Saint-Laurent comme un édifice ayant une nef composée de trois vaisseaux et de quatre ou cinq travées.

Le projet d'aménagement de ce secteur dit « square des bénédictins » est donc envisagé de façon indépendante du reste du prieuré. De plus, ce qui est conservé de l'ancien jardin avec un axe de circulation nord-sud sépare topographiquement l'église SaintLaurent de la prieurale Notre-Dame.

\section{Une nouvelle problématique}

16 Le dégagement du chevet de l'église Saint-Laurent avait permis de retrouver de nombreux blocs architecturaux, appartenant à son élévation, qui s'étaient effondrés à l'intérieur du bâtiment. Dans le cadre du programme d'aménagement du square, un projet d'anastylose, c'est-à-dire le remontage des murs à partir des blocs découverts in situ, a nécessité l'inventaire de 2000 pierres taillées et sculptées. Le travail d'analyse a montré la nécessité de pousser plus à fond l'étude architecturale de cet édifice et de mener une étude comparative pour son plan et son élévation.

Il est alors apparu clairement que cette construction pouvait être contemporaine de l'église Notre-Dame et non antérieure. Elle n'était donc pas à appréhender comme indépendante du reste du prieuré mais devait être restituée dans l'ensemble du site, en lien avec les alentours, l'église Notre-Dame et les bâtiments monastiques, mais aussi la ville.

Cette nouvelle hypothèse d'interprétation sur l'identité de l'église Saint-Laurent a posé de nombreuses questions :

- quelles étaient les dimensions de la nef, tant en longueur qu'en largeur?

- quels étaient ses liens avec le reste du prieuré ?

- comment comprendre la topographie médiévale de ce secteur du prieuré?

- quelle était la fonction de cette église annexe?

Cela remettait en cause la compréhension du site et donc tout l'aménagement, notamment les questions de déambulation dans ce secteur.

Il n'a pas été facile à l'architecte en chef chargé du projet, qui travaillait depuis plus de 10 ans sur l'idée d'un ensemble monastique séparé du reste des constructions, de basculer dans une problématique inverse !

21 Pas plus d'ailleurs pour les habitants qui avaient l'habitude de passer entre les deux chevets, chemin qui était le plus direct, de les inviter à faire un détour pour passer audelà du chevet de Saint-Laurent.

Il était donc important de pouvoir vérifier cette nouvelle hypothèse en mettant en place tout d'abord des sondages avant d'engager une campagne de fouille.

Entre temps, les travaux d'aménagement pour une aire de promenade au chevet de Notre-Dame avaient commencé. Les sondages puis la fouille des niveaux archéologiques mettent au jour de très nombreuses sépultures témoignant d'une occupation dense de l'espace et une longue galerie de $3 \mathrm{~m}$ de large datant du XIII siècle. Cette découverte renforce l'idée que l'église Saint-Laurent n'est pas à séparer du reste du prieuré.

Signalons que l'intervention n'a pas été une fouille complète du secteur mais ne concernait que les niveaux touchés par le projet prévu à l'époque. Dix ans plus tard, à l'occasion du nouvel aménagement, il a fallu reprendre la fouille. 

l'aménagement général du square et de l'assainissement des bâtiments conventuels, a permis d'attester que le chevet était bien en lien avec le reste du prieuré : ouverture dans le mur de la salle du chapitre; dégagement des murs gouttereaux jusqu'à sa proximité; mêmes mises en œuvre des constructions; mise au jour de différents niveaux de sols et de tout un système de circulation, modifiés au fil du temps; utilisation ininterrompue de ce lieu jusqu'à sa disparition au Xvi ${ }^{\mathrm{e}}$ siècle.

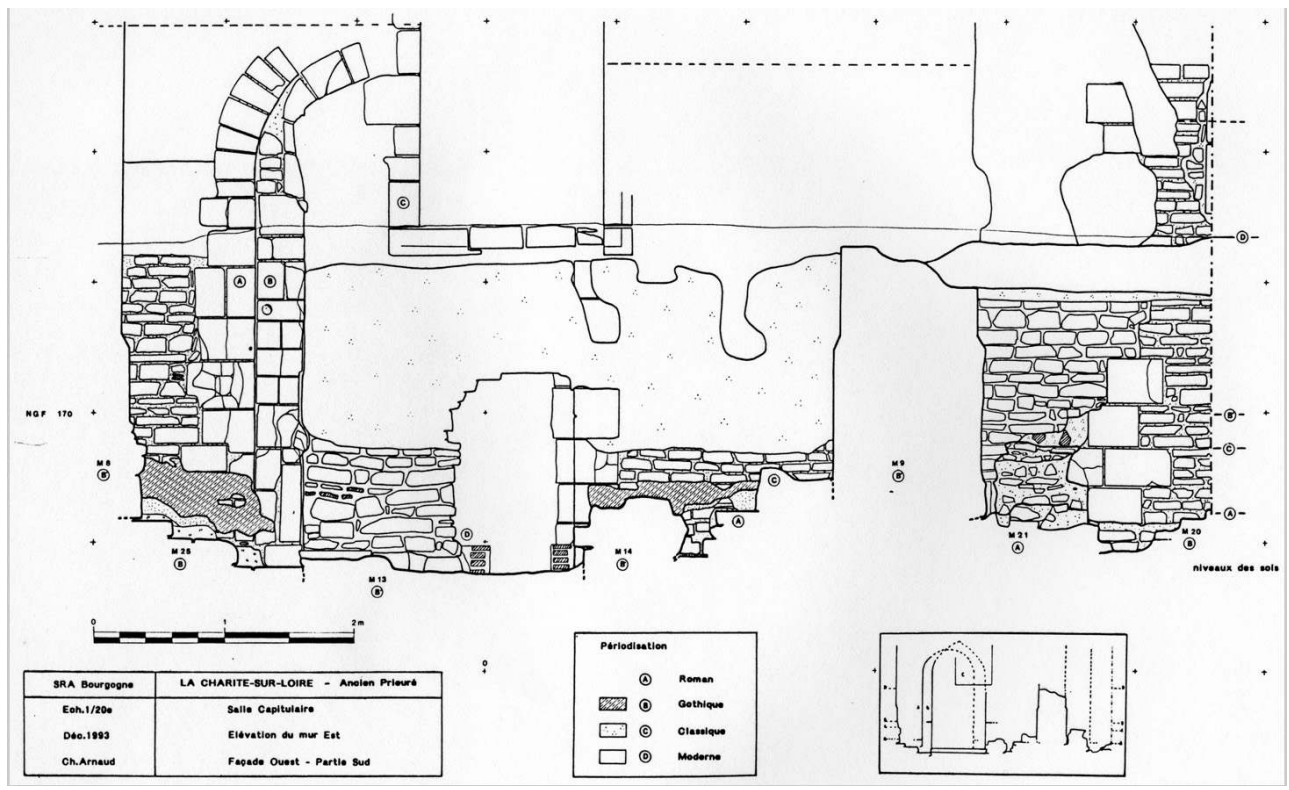

Fig. 2 - Élévation extérieure du mur est de la salle du chapitre (Ch. Arnaud).

\section{Fonction et évolution de l'église Saint-Laurent}

Les recherches archéologiques ont montré que le plan de l'ensemble de ce monastère clunisien a été conçu dès l'origine de façon très développée. Comment comprendre qu'au $\mathrm{XI}^{\mathrm{e}}$ siècle, lorsque les moines viennent s'établir en cet endroit, ils construisent au moins deux églises en même temps que les bâtiments conventuels?

Le développement de ce plan peut se comprendre par l'intégration, dans la topographie du monastère, des nouveautés liturgiques clunisiennes qui prennent une nouvelle

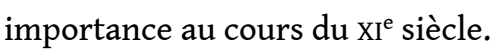

À Cluny, sont conservés des livres, appelés coutumiers, qui précisent les rites de la liturgie monastique clunisienne. Ainsi sait-on que les moines se rendaient en procession de l'église abbatiale à l'église annexe Notre-Dame, correspondant topographiquement à l'église Saint-Laurent, pour y célébrer l'office des saints et des défunts et ce au moins deux fois par jour?

On peut supposer qu'il en était de même à La Charité-sur-Loire: les textes nous apprennent que c'est dans cette église de tous les saints, dite de Saint-Laurent, que l'évêque Geoffroy de Champallement, un des acteurs de la fondation du monastère de La Charité, est inhumé en 1071. D'autre part, cette fonction de commémoration est attestée par la présence de dalles funéraires découvertes sur le site et datant du XIV siècle. 


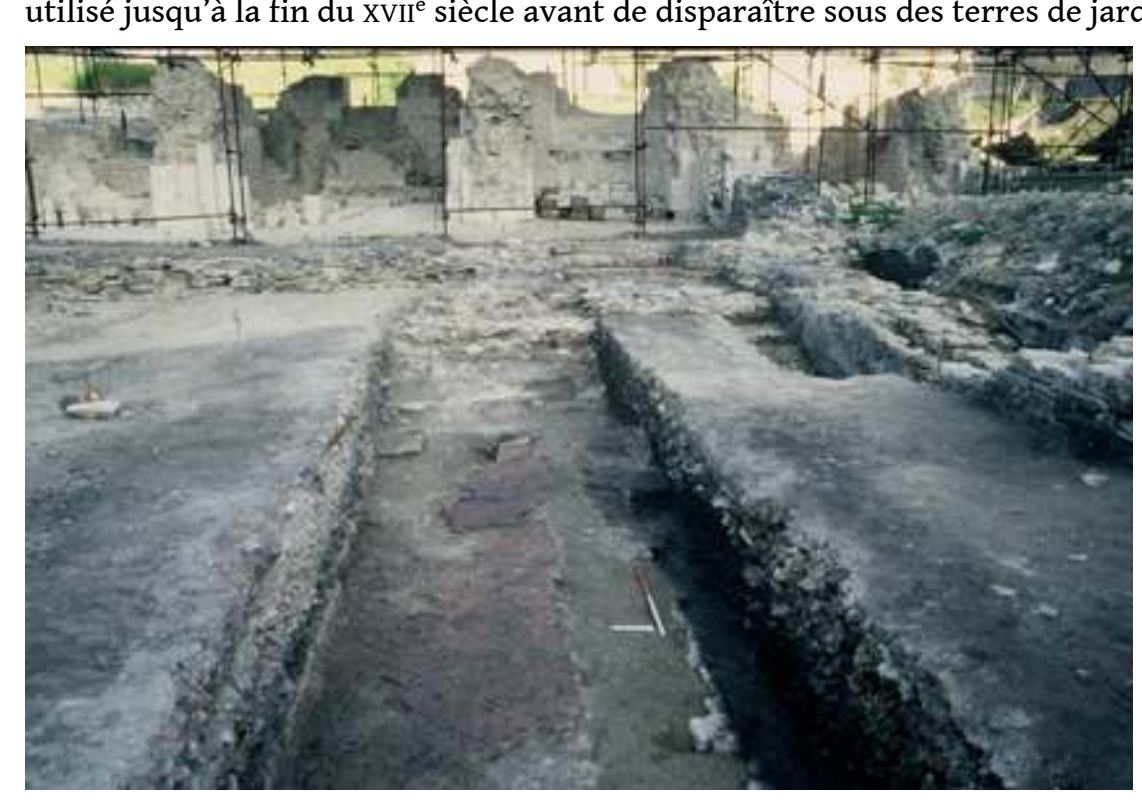

Fig. 3 - Vue de la nef de Saint-Laurent depuis la salle du chapitre vers le chevet (Ch. Arnaud).

Les travaux archéologiques ont permis également de cerner l'évolution des aménagements de l'église Saint-Laurent: un chevet à trois absides échelonnées, un transept non saillant (ou travée droite) et une nef unique.

Dans un premier état, l'accès au chœur, légèrement plus surélevé, se faisait par deux escaliers latéraux alors qu'au centre, une maçonnerie avançait dans la nef, sans doute un podium. Différents paliers scandaient la nef en raison de la topographie du terrain ; l'accès à la nef de cette église se faisait soit par la salle du chapitre, à l'extrémité ouest, soit par le cimetière, du côté sud.

32 À partir du début du XIII ${ }^{\mathrm{e}}$ siècle, d'importantes transformations modifient la circulation intérieure et extérieure à l'église: l'accès au chœur s'effectue par un escalier se développant sur toute la largeur de la nef; la porte sud est déplacée lors de la construction d'une large galerie d'accès.

Vers la fin $\mathrm{du} \mathrm{XV}^{\mathrm{e}}$ siècle, sont installées d'importantes stalles et l'ensemble du bâtiment est restauré.

Suite à l'incendie de 1559, la nef est en partie abandonnée alors que le chevet est encore utilisé jusqu'à la fin du XVII ${ }^{\mathrm{e}}$ siècle avant de disparaître sous des terres de jardin.

\section{Conclusion}




\section{BIBLIOGRAPHIE}

Pour toutes références archivistiques et bibliographiques, cf. Ch. ARNAUD et M.-J. GARNICHE, « Le prieuré clunisien de La Charité-sur-Loire. État de la question ", in Bulletin de la Société nivernaise des lettres, sciences et arts, 44 (1995) p. 63-94.

ARNAUD Ch., "La Charité-sur-Loire », in Les églises de l'ancien diocèse d'Auxerre, SSHNY, 2009, p. $695-724$

INDEX

Index géographique : France/La Charité-sur-Loire 